Research article

Open Access

\title{
Determination of HER2 phosphorylation at tyrosine 1221/1222 improves prediction of poor survival for breast cancer patients with hormone receptor-positive tumors
}

\author{
Thomas Frogne1, Anne-Vibeke Laenkholm², Maria B Lyng ${ }^{3}$, Katrine Lütken Henriksen ${ }^{1}$ and \\ Anne E Lykkesfeldt ${ }^{1}$
}

\begin{abstract}
1Department of Tumor Endocrinology, Institute of Cancer Biology, Danish Cancer Society, Strandboulevarden 49, DK-2100 Copenhagen, Denmark 2Department of Pathology, Odense University Hospital, Winsloewparken 15, 5000 Odense C, Denmark

${ }_{3}^{3}$ Medical Biotechnology Center, University of Southern Denmark, J.B. Winsloewsvej 25.3, 5000 Odense C, Denmark

Corresponding author: Anne E Lykkesfeldt, al@cancer.dk
\end{abstract}

Received: 5 Nov 2008 Revisions requested: 21 Nov 2008 Revisions received: 29 Jan 2009 Accepted: 24 Feb 2009 Published: 24 Feb 2009

Breast Cancer Research 2009, 11:R11 (doi:10.1186/bcr2230)

This article is online at: http://breast-cancer-research.com/content/11/1/R11

(c) 2009 Frogne et al.; licensee BioMed Central Ltd.

This is an open access article distributed under the terms of the Creative Commons Attribution License (http://creativecommons.org/licenses/by/2.0), which permits unrestricted use, distribution, and reproduction in any medium, provided the original work is properly cited.

\begin{abstract}
Introduction High expression of total HER2 protein confers poor prognosis for breast cancer patients. HER2 is a member of the HER family consisting of four receptors, HER1 to HER4. HER receptor activity is regulated by a variety of mechanisms, and phosphorylation of the C-terminal part of the HER receptors is a marker for active signaling. The importance of phosphorylation and thereby activation of the HER1 to HER4 receptors, however, has not been investigated concomitantly in breast tumors. In the present study we examined the importance of active HER signaling in breast tumor biopsies and paired metastases, by evaluating the expression of phosphorylated HER1, HER2, HER3, Erk, Akt and the total level of HER4 and HER2.

Methods Immunohistochemical analysis was performed on 268 primary breast tumors and 30 paired metastatic lesions from postmenopausal women with hormone receptor-positive breast tumors, who had received adjuvant tamoxifen therapy. The observed protein expression levels were analyzed for coexpression, for correlation to clinicopathological parameters and for prognostic value in relation to disease-free survival and overall survival. Lastly, the difference between protein levels in primary tumors versus metastasis was evaluated.
\end{abstract}

Results In the primary tumors, $8 \%, 18 \%, 14 \%$ and $15 \%$ of cases were scored positive for total HER2, pHER1, pHER2 and pHER3 expression, respectively. HER4 was expressed with strong intensity in $68 \%$ and at moderate intensity in $29 \%$ of cases. The activated forms of Akt and Erk were quite uniformly expressed in the categories; negative, moderate or strong. In univariate analysis, expression of total HER2, pHER1, pHER2 and pHER3 was significantly associated with poor disease-free survival. Strong HER4 expression was associated with prolonged disease-free as well as with overall survival. Expression of pAkt and pErk was not correlated with survival. In multivariate analysis, pHER2 expression was clearly an independent marker for poor disease-free survival and overall survival when tested against tumor size, tumor grade, nodal status and HER2. Lastly, comparison of HER receptor expression in metastatic versus primary tumors showed a significant increase in expression of pHER1 and pHER3 in the metastases.

Conclusions In hormone receptor-positive breast cancer, determination of pHER2 yields additional prognostic information about poor prognosis compared with the current clinical standard for measuring HER2.

\section{Introduction}

The epidermal growth factor receptor family constitutes four members: HER1 (EGFR/ErbB1), HER2 (ErbB2), HER3 (ErbB3) and HER4 (ErbB4). The four receptors are activated by binding of numerous ligands, which leads to heterodimerization or homodimerization and subsequent phosphorylation of specific tyrosine residues in the intracellular region. This activation by phosphorylation results in regulation of a variety of cellular processes including cell proliferation and survival. Much evidence suggests that the kinases Akt and Erk mediate a substantial part of the HER signaling [1]. 
In breast cancer, excellent work has been done to investigate the significance of HER signaling for prognosis, both by characterization of the HER dimers [2] as well as of the ligands [3]. The most studied receptors in breast cancer are HER1 and HER2; overexpression of HER2 generally occurs in about $20 \%$ of breast carcinomas, and is more frequent in estrogen receptor-negative than in estrogen receptor-positive cases [4]. HER1 and HER2 overexpression is generally associated with more aggressive tumors and poor prognosis [2,5-7]. The importance of HER3 has been less investigated, but from in vitro studies it is becoming clear that HER3 is involved in tumor growth and also in resistance to both endocrine and HER-directed treatment [8-10]. Several recent clinical studies have found HER3 to correlate with adverse clinicopathological properties $[2,11,12]$. Association with better prognosis, however, has also been reported [13,14]. The role of HER4 in breast cancer has also been investigated - and in estrogen receptor-positive breast cancer cell lines, HER4 is often found to mediate growth inhibition and cell differentiation $[15,16]$. Analysis of tumor biopsies generally shows that expression of HER4 is associated with prolonged survival $[2,6,14,17,18]$.

In the clinical setting today, only three protein biomarkers are used; estrogen receptor, progesterone receptor and HER2. The golden standard for determination of HER2 positivity is evaluation by immunohistochemistry (IHC); and for IHC equivocal cases, this is followed by fluorescence in situ hybridization (FISH) analysis for determination of gene amplification. Patients with HER2-positive tumors are candidates for treatment with the monoclonal anti-HER2 antibody trastuzumab. Numerous other approaches, however, are currently being evaluated in clinical trials for their ability to inhibit HER1 and/ or HER2 or all four HER receptors [19]. It is therefore important to have good quality biomarkers to ensure that the patient is offered optimal treatment.

Our intention with the present study was to concomitantly evaluate the activation status of HER1, HER2 and HER3 and to determine their importance in hormone receptor-positive breast cancer. We hereby aimed to find new and superior biomarkers for detection of poor prognosis, and also to suggest new markers for future investigations regarding prediction of benefit from endocrine and/or anti-HER therapy. We therefore measured the levels of HER1, HER2 and HER3, which were phosphorylated at tyrosine sites described to be important for activation of the receptors [20], as well as the levels of the activated form of the downstream target kinases Erk and Akt. We were also interested in determining the amount of pHER4, but we were not able to find a commercially available anti-pHER4 antibody that was pHER4 specific and applicable in IHC analysis. Instead, we measured total HER4 expression using an antibody against the $\mathrm{C}$-terminal region. Overall, we report the analysis of phosphorylated HER1, HER2, HER3, Erk, and Akt as well as total HER2 and HER4 expression levels in 268 hormone receptor-positive primary tumors and 30 corresponding metastases from postmenopausal women treated adjuvantly with tamoxifen.

\section{Materials and methods Patients}

A total of 268 postmenopausal patients were used for this study, all of which underwent surgery for primary invasive breast cancer between 1989 and 2001 at Odense University Hospital. Patients were classified as high risk, according to age $(<75$ years) and/or tumor size $(>2 \mathrm{~cm})$ and/or Bloom and Richardson grade $(\geq 2)$ and/or positive nodal status. The highrisk patients received tamoxifen as the first-line adjuvant treatment and all, except a single case, were positive for either estrogen receptor and/or progesterone receptor. Owing to side effects, 25 patients were subsequently crossed over from tamoxifen to megace or letrozole. The only other criterion for entering this study was that both paraffin-embedded and frozen tumor tissue was present.

The standard clinicopathological parameters are presented in Table 1. HER2 positivity was determined according to current standardized guidelines for HER2 testing; that is, IHC followed by FISH analysis for cases scoring 2+. The analysis was performed with the HercepTest ${ }^{\mathrm{TM}}$ for $\mathrm{IHC}$ and with pharmDx ${ }^{\mathrm{TM}}$ for FISH (both from DAKO A/S, Glostrup, Denmark).

The total number of malignancy graded tumors was only 228; this was due to tumors of lobular subtype and tumors of rare subtypes, which were not graded. Table 2 presents the patient characteristics and treatment characteristics. It should also be mentioned that only a single patient experienced relapse while receiving tamoxifen.

The analyses on the clinical material have been approved by the local ethics committee for Region South Denmark, S-VF20040064.

\section{Tumor material and construction of tissue microarrays}

Archival formalin-fixed and paraffin-embedded primary tumor tissue was used to generate tissue microarrays comprised of two $2 \mathrm{~mm}$ cores from each tumor, as previously described [21]. For positive controls, we included tissue cores of epidermis (pHER1), striated muscle (HER4) and of the MCF-7 cell line, which had been treated with the HER3/4 family ligand heregulin $1 \beta$ (recombinant heregulin 1 $\beta$, 396-HB/CF; R\&D Systems Europe Ltd, Abingdon, UK; pHER2, pHER3, HER4). Cores on each tissue miroarray were therefore known to express a high amount of the six proteins evaluated in this study. Negative controls were obtained by omitting the primary antibody.

Tumor metastases were available from 30 patients and were mostly distant metastases; for example, from skin (six patients), bone (four patients), bone marrow (three patients), pleural effusions (three patients), soft tissue (four patients), 
Table 1

\section{Clinicopathological parameters}

\begin{tabular}{|c|c|c|}
\hline Parameter & $n$ & $\%$ \\
\hline \multicolumn{3}{|l|}{ World Health Organization diagnosis } \\
\hline Invasive ductal & 230 & 86 \\
\hline Invasive lobular & 32 & 12 \\
\hline Ductal with ductal carcinoma in situ & 2 & 1 \\
\hline Unknown & 4 & 1 \\
\hline Total & 268 & \\
\hline \multicolumn{3}{|l|}{ Tumor grade } \\
\hline 1 & 62 & 27 \\
\hline 2 & 103 & 45 \\
\hline 3 & 63 & 28 \\
\hline Total & 228 & \\
\hline \multicolumn{3}{|l|}{ Tumor size } \\
\hline$<2 \mathrm{~cm}$ & 85 & 32 \\
\hline 2 to $5 \mathrm{~cm}$ & 161 & 60 \\
\hline$>5 \mathrm{~cm}$ & 22 & 8 \\
\hline Total & 268 & \\
\hline \multicolumn{3}{|l|}{ Nodal status } \\
\hline 0 & 22 & 8 \\
\hline 1 to 3 & 149 & 56 \\
\hline$\geq 4$ & 97 & 36 \\
\hline Total & 268 & \\
\hline \multicolumn{3}{|c|}{ Receptor status (estrogen receptor or progesterone receptor) } \\
\hline Negative & 1 & 0 \\
\hline Positive & 267 & 100 \\
\hline Total & 268 & \\
\hline \multicolumn{3}{|l|}{ HER2 } \\
\hline Negative & 246 & 92 \\
\hline Positive & 22 & 8 \\
\hline Total & 268 & \\
\hline \multicolumn{3}{|l|}{ Recurrence } \\
\hline No & 179 & 67 \\
\hline Yes & 89 & 33 \\
\hline Total & 268 & \\
\hline \multicolumn{3}{|l|}{ Death } \\
\hline No & 146 & 54 \\
\hline Yes & 122 & 46 \\
\hline Total & 268 & \\
\hline
\end{tabular}

Tumor grade was scored according to Bloom and Richardson. HER2 was analyzed by standard testing (that is, the HercepTest ${ }^{\mathrm{TM}}$ ), followed by fluorescence in situ hybridization analysis of the cases scoring $2+$. 
Table 2

\begin{tabular}{lll}
\hline \multicolumn{3}{l}{ Patient and treatment characteristics } \\
\hline Median (years) & Range (years) \\
\hline Age & 61 & 48 to 74 \\
Time on tamoxifen & 1.8 & 0.4 to 6.0 \\
Time to recurrence & 3.1 & 0.12 to 16.2 \\
Time to death & 5.9 & 0.26 to 15.7 \\
Time of follow up & 12.4 & 6.3 to 19.2 \\
& & \\
\hline
\end{tabular}

peripheral lymph nodes (two patients) and visceral organs (eight patients).

\section{Immunohistochemistry}

Serial sections of $5 \mu \mathrm{m}$ thickness were cut from the tissue microarrays, dewaxed and rehydrated. To block endogenous peroxidase, the slides were incubated in $1.5 \%$ hydrogen peroxide in Tris-buffered saline for 10 minutes.

Antigen retrieval was performed by three methods. When staining for pHER1, pHER2, pAkt or pErk, heat-induced epitope retrieval was performed by microwave oven for 15 minutes in TEG buffer (10 mM Tris, $0.5 \mathrm{mM}$ ethylene glycol tetraacetic acid, pH 9); for pHER3, the TEG buffer was replaced with $1 \mathrm{mM}$ ethylenediamine tetraacetic acid at $\mathrm{pH} 8$. Antigen retrieval for the HER4 staining was done by a 15minute protease treatment.

Incubation with primary antibody for 60 minutes at room temperature was followed by detection of the primary antibody using the Advance ${ }^{\mathrm{TM}}$ HRP system (DAKO). The applied chromogen was 3,3'-diaminobenzidine and all stainings were performed in the Autostainer Plus Link Instrument (DAKO). After washing, the slides were counterstained with Meyer's hematoxylin for 30 seconds. The following antibodies were used: pHER11173 (dilution factor 1:200, catalog number 4407), pHER21221/1222 (dilution factor 1:200, catalog number 2243), pHER31289 (dilution factor 1:100, catalog number 4791), HER4c-terminal (dilution factor 1:250, catalog number RB9045), pErk202/204 (dilution factor 1:1000, catalog number 4376) and $\mathrm{pAkt}^{473}$ (dilution factor 1:200, catalog number 3787). The HER4 antibody was from Thermo Fisher Scientific (Fremont, CA, USA), whereas all other antibodies were from Cell Signaling Technology (Danvers, MA, USA), which in the datasheets for the antibodies against the individual HER receptor demonstrated no cross-reaction to the other HER receptors.

\section{Evaluation of immunohistochemistry data}

Two different scoring methods were used in the present study. The markers with predominantly membrane staining (HER2, pHER1, pHER2 and pHER3) were scored according to the
HercepTest ${ }^{\mathrm{TM}}$ guidelines. For markers with predominantly cytoplasmic staining (HER4, pAkt and pErk), the overall staining intensity was scored.

In the HercepTest ${ }^{\mathrm{TM}}$ : $0=$ no staining or membranous staining in $<10 \%$ of invasive tumor cells; $1=$ faint or barely perceptible membranous staining in $>10 \%$ of invasive tumor cells; and 2 $=$ moderate and $3=$ strong complete membranous staining in $>10 \%$ of invasive tumor cells, respectively.

The overall staining intensity (that is, staining of membrane, cytoplasm and/or nucleus) was scored if $>10 \%$ of the tumor cells were positive. We used a scale from 0 to $2: 0=$ negative, $1=$ moderate and $2=$ strong staining intensity.

Furthermore, nuclear reactivity of all stainings was scored as either negative or positive, again with a cutoff value of $10 \%$ positive tumor cells.

TF scored all primary tumors and A-VL scored all metastases as well as $25 \%$ of the primary tumors. The scores from the two observers were not different by McNemar's test, and the kappa statistic ranged from 0.8 to 1.0 . The six investigated proteins were scored in two cores from each tumor in approximately $90 \%$ of cases, whereas the remaining cases were scored from a single core. In the rare cases where the two score values differed by 2 , the highest score value was used [21].

\section{Statistical analysis}

Spearman correlation coefficients and Fisher's exact test were used to determine significant associations between the expression levels of the investigated proteins as well as to the clinicopathological parameters. Survival curves were generated by Kaplan-Meier estimates followed by the log-rank test. The Cox proportional hazards model was used to evaluate the prognostic value of the markers both alone (univariate) and in combination (multivariate) with the current standard markers: tumor grade, tumor size, nodal status and HER2. Lastly, the Sign test was used to evaluate differences between the protein expression levels of primary and metastatic tumors. All tests were two sided and $P<0.05$ was considered significant. SAS 9.1 software (SAS Institute Inc., Cary, NC, USA) was used for all analyses.

\section{Results}

Expression levels of phosphorylated HER1, HER2, HER3, Erk and Akt and total HER4

Membrane reactivity of pHER1, pHER2 and pHER3 was evaluated using the HercepTest ${ }^{\mathrm{TM}}$ score method, as described in Materials and methods. Expression of pHER1 was evaluated in 264 cases and 18\% had a detectable membrane staining, of which the majority were weak (score 1) and a few showed moderate intensity (score 2). No tumors with strong (score 3) pHER1 membrane staining were observed. Expression of 
pHER2 was analyzed in 264 tumors and membrane staining with scores 1, 2 and 3 was recorded in $27 \%, 11 \%$ and $4 \%$ of cases, respectively. The moderate and strong pHER2 membrane reactivity was very often accompanied by cytoplasmic staining. We evaluated pHER3 expression in 261 cases and the strongest expression was found in the membrane and nucleus, but staining was also observed in the cytoplasm. Weak membrane staining was observed in $11 \%$ of cases, and only $3 \%$ and $1 \%$ were recorded with the scores 2 and 3 , respectively. Interestingly, we also observed substantial nuclear pHER3 staining in 10\% of the 261 cases. Expression of HER4 was evaluated in 259 cases and the staining was predominantly cytoplasmic, but most often in conjunction with a variable amount of membrane reactivity. We did not observe nuclear reactivity with this HER4 antibody. Owing to the considerable amount of cytoplasmic reaction, the HER4 staining was not scored by the HercepTest ${ }^{\mathrm{TM}}$ but by the overall intensity, grouped as either negative (score 0), moderate (score 1) or strong (score 2). The moderate intensity was observed in $29 \%$ of cases, $68 \%$ showed the strong intensity and only $3 \%$ were negative. Figure 1 shows representative pictures of the observed staining patterns of the four HER receptors.

The same overall intensity score was used to record the expression level of pErk (256 cases) and of pAkt (261 cases). Both the pErk and pAkt stainings were cytoplasmic and nuclear. The pErk expression was quite heterogeneous especially in the nucleus, whereas pAkt was more homogeneous and the same intensity was usually observed in both the cytoplasm and nucleus. We observed a fairly even distribution of cases in the three categories, with a few more pErk-negative tumors and slightly more tumors expressing a moderate level of pAkt. Based on these initial frequency data, we decided to assess pHER1 and pHER3 as either negative (score 0) or positive (scores 1, 2 and 3), to assess pHER2 as either negative (score 0), weak (score 1) or strong (scores 2 and 3), and to assess HER4 as either low (scores 0 and 1) or high (score 2). The pErk and pAkt scores were analyzed in the original categories.
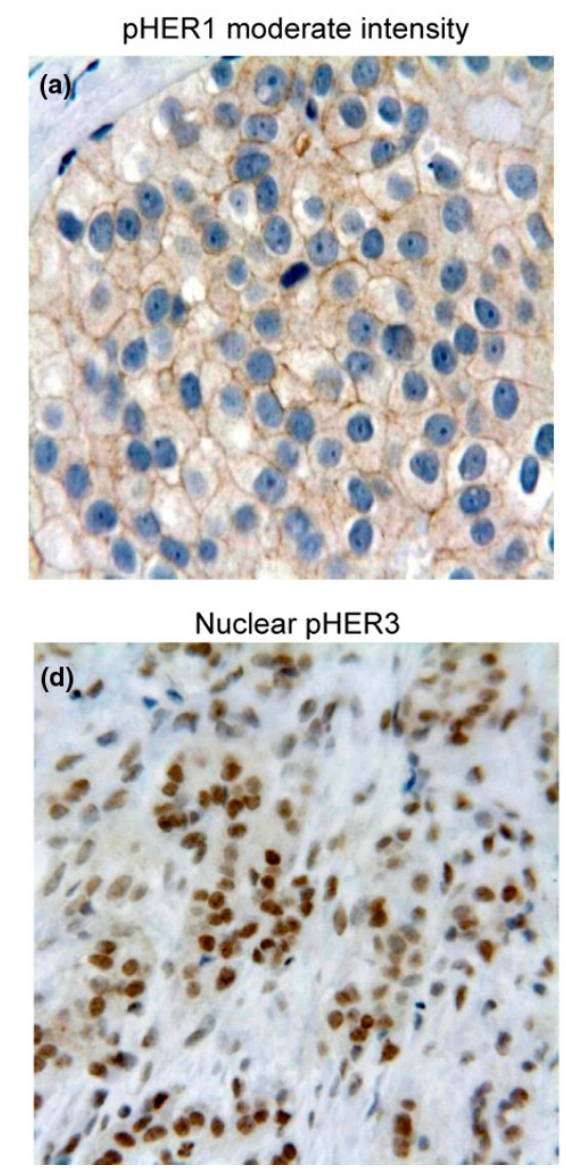

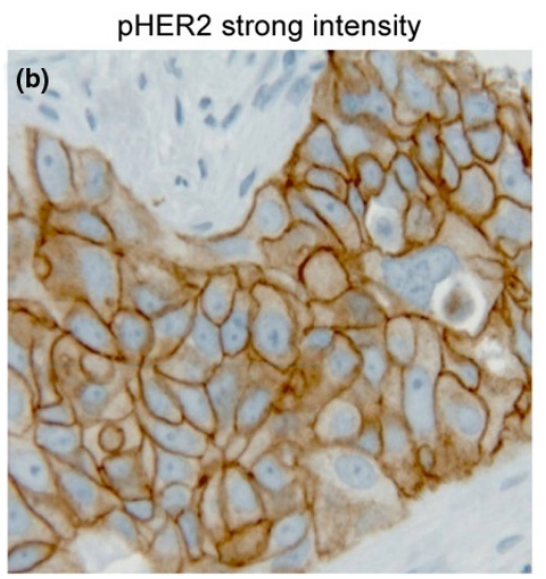

HER4 moderate intensity

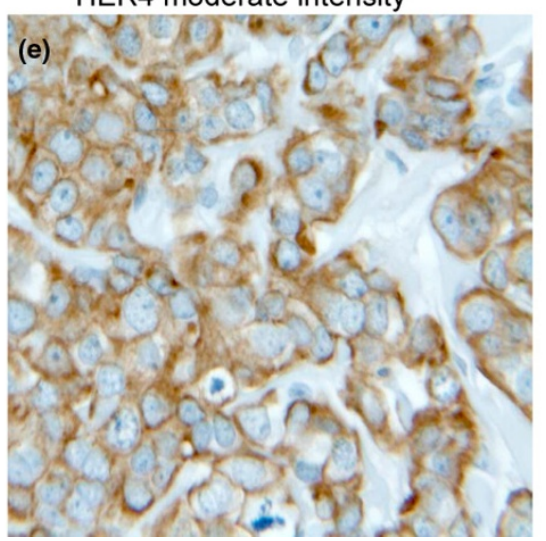

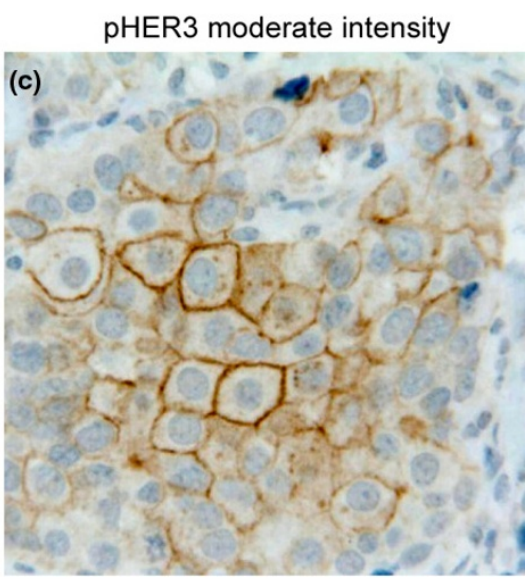

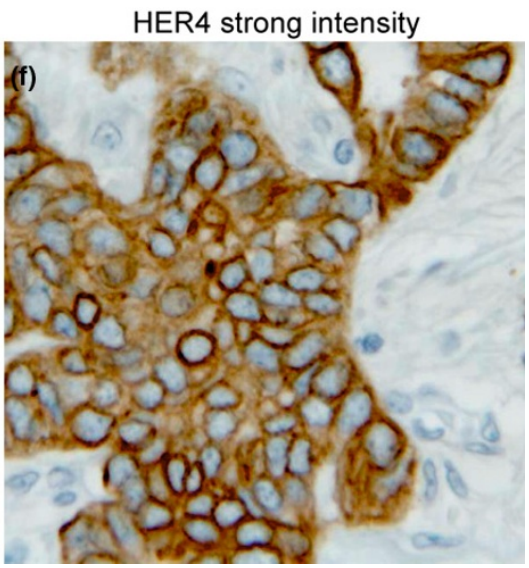

Immunohistochemical staining of primary breast tumors. (a) Moderate-intensity membranous pHER1. (b) Strong-intensity membranous pHER2. (c) Moderate-intensity membranous pHER3. (d) Nuclear pHER3. (e) Moderate HER4 intensity. (f) Strong HER4 intensity. 


\section{Correlations between protein expression levels}

Table 3 presents the protein expression levels and their frequencies in relation to each other. Fisher's exact test was used to determine significant correlations between the investigated protein expression levels. We found strong positive correlation between membrane expression of HER2, pHER2 and pHER3. Briefly, $68 \%$ of the HER2-positive cases also showed strong pHER2 expression, while $28 \%$ of HER2-positive cases displayed weak pHER2 expression. Only a single patient therefore had a HER2-positive tumor without concurrent expression of pHER2. Conversely, only 39\% of the strong-expressing pHER2 cases were found to be HER2-positive. Of the remaining HER2-negative and pHER2-positive cases (23 cases, $61 \%), 15$ were scored 0 with the HercepTest ${ }^{\mathrm{TM}}$, six were scored +1 , and two were scored +2 and without amplification. The HER2-negative cases with strong pHER2 expression were in $82 \%$ of cases expressed concomitant with pHER1 and/or pHER3. pHER2-positive cases that were scored HER2-positive expressed pHER1 and/or pHER3 in 74\% of cases. The activated HER2 is therefore generally coexpressed with at least one other activated HER receptor that has the capacity to function as a dimerization partner. We used serial sections, and Figure 2 is a representative picture showing co-expression of all three phosphorylated receptors in the same tumor cells. Lastly, pHER2, but not HER2, was negatively correlated to HER4 expression.

Expression of the activated form of the intracellular kinases Akt and Erk was highly correlated, and pAkt was also associated with expression of pHER2 and pHER3 - and perhaps also with pHER1 $(P=0.057)$. No significant correlations between pErk and the HER receptors were observed.

\section{Associations between protein expression levels and standard clinicopathological parameters}

Table 4 presents the classification of the observed protein expression levels in relation to the parameters of tumor grade, tumor size, nodal status, recurrence and death. From these distributions we used Fisher's exact test to analyze for potential significant correlations. Expression of HER2, pHER1 and pHER2 was found to correlate with recurrence. For HER2 and pHER2 we also found a positive correlation with high tumor grade, but interestingly only pHER2 was associated with the event of death. In contrast, we observed negative correlations between HER4 expression and grade, recurrence and death. Lastly, pAkt and pErk were not associated with the standard clinicopathological parameters.

\section{Kaplan-Meier plots for the HER receptors in relation to disease-free survival}

Figure 3 shows the Kaplan-Meier plots of pHER1, pHER2, pHER3 and HER4 expression levels in relation to disease-free survival. All four markers were found to be significantly associated with disease-free survival. Most of all, strong but not weak expression of pHER2 was highly associated with decreased disease-free survival. Likewise, although not as significant, expression of pHER1 and pHER3 was also found to correlate with reduced disease-free survival. In contrast, a high amount of HER4 was clearly associated with prolonged disease-free survival.

\section{Cox univariate and multivariate regression analysis of variables in relation to disease-free survival and overall survival}

Based on the Kaplan-Meier plots we performed a Cox regression with the variables analyzed in the following categories: tumor grade ( 1 and 2 vs. 3), tumor size ( $<25 \mathrm{~mm}$ vs. $\geq 25 \mathrm{~mm}$ ),

Figure 2

Membranous pHER1

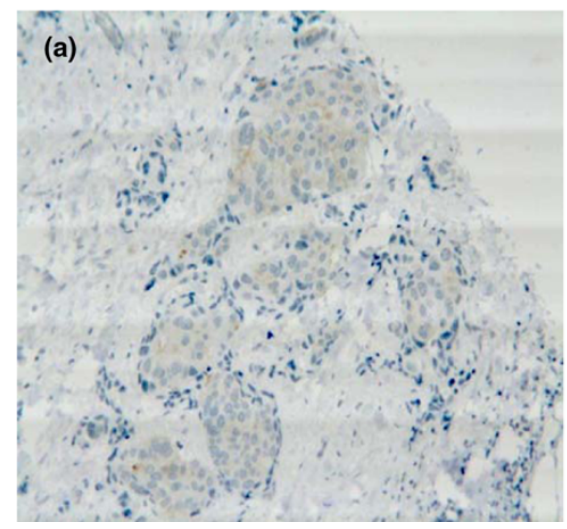

Membranous pHER2

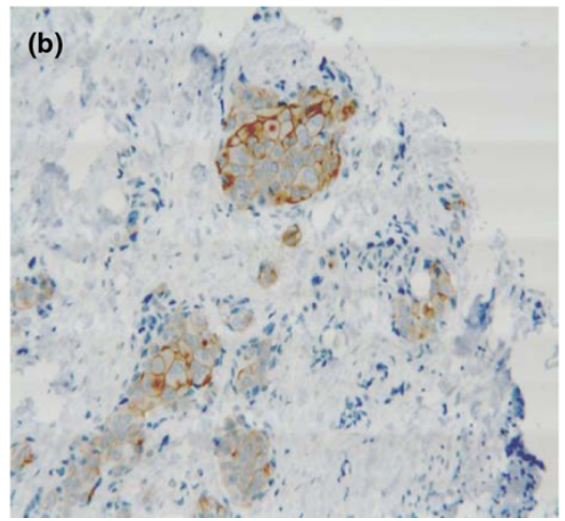

Membranous pHER3

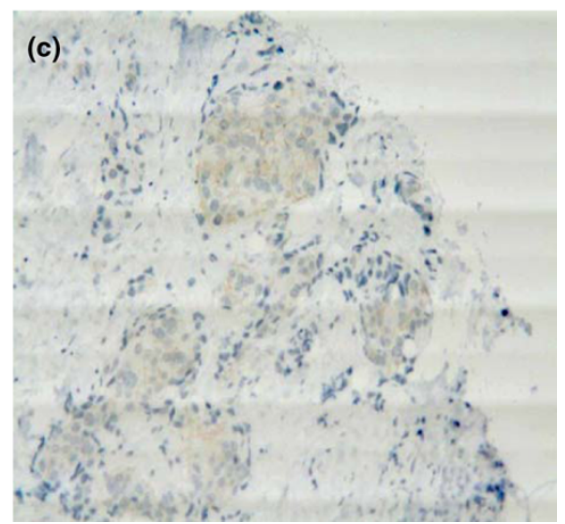

Immunohistochemical staining of primary breast tumor positive for activated forms of HER1, HER2 and HER3. Immunohistochemical staining of serial sections of a primary breast tumor showing that the same cells are positive for the activated forms of HER1, HER2 and HER3. (a) Membranous pHER1. (b) Membranous pHER2. (c) Membranous pHER3. 


\begin{tabular}{|c|c|c|c|c|c|c|c|c|c|c|c|c|c|c|}
\hline & \multicolumn{2}{|c|}{ HER2 } & \multicolumn{2}{|c|}{ pHER1 } & \multicolumn{3}{|c|}{ pHER2 } & \multicolumn{2}{|c|}{ pHER3 } & \multicolumn{2}{|c|}{ HER4 } & \multicolumn{3}{|c|}{ pErk } \\
\hline & $(-)$ & $(+)$ & $(-)$ & $(+)$ & $(-)$ & Weak & Strong & $(-)$ & $(+)$ & Low & High & $(-)$ & Moderate & Strong \\
\hline \multicolumn{15}{|l|}{ pHER1 } \\
\hline Negative & 202 & 15 & & & & & & & & & & & & \\
\hline Positive & 40 & 7 & & & & & & & & & & & & \\
\hline$P$ value & \multicolumn{2}{|c|}{0.083} & & & & & & & & & & & & \\
\hline \multicolumn{15}{|l|}{ pHER2 } \\
\hline Negative & 154 & 1 & 148 & 7 & & & & & & & & & & \\
\hline Weak & 65 & 6 & 55 & 15 & & & & & & & & & & \\
\hline Strong & 23 & 15 & 13 & 25 & & & & & & & & & & \\
\hline$P$ value & \multicolumn{2}{|c|}{$<0.0001(+)$} & \multicolumn{2}{|c|}{$<0.0001(+)$} & & & & & & & & & & \\
\hline \multicolumn{15}{|l|}{ pHER3 } \\
\hline Negative & 211 & 10 & 199 & 21 & 144 & 61 & 14 & & & & & & & \\
\hline Positive & 28 & 12 & 13 & 26 & 6 & 10 & 24 & & & & & & & \\
\hline$P$ value & \multicolumn{2}{|c|}{$<0.0001(+)$} & \multicolumn{2}{|c|}{$<0.0001(+)$} & \multicolumn{2}{|c|}{$<0.0001(+)$} & & & & & & & & \\
\hline \multicolumn{15}{|l|}{ HER4 } \\
\hline Low & 75 & 10 & 69 & 16 & 49 & 16 & 19 & 71 & 13 & & & & & \\
\hline High & 163 & 12 & 142 & 31 & 102 & 53 & 19 & 145 & 27 & & & & & \\
\hline$P$ value & \multicolumn{2}{|c|}{0.234} & \multicolumn{2}{|c|}{0.865} & \multicolumn{2}{|c|}{$0.021(-)$} & & \multicolumn{2}{|c|}{1.000} & & & & & \\
\hline \multicolumn{15}{|l|}{ pErk } \\
\hline Negative & 103 & 10 & 97 & 16 & 70 & 25 & 17 & 99 & 12 & 44 & 65 & & & \\
\hline Moderate & 56 & 5 & 47 & 14 & 31 & 21 & 9 & 52 & 9 & 17 & 42 & & & \\
\hline Strong & 75 & 7 & 65 & 15 & 46 & 24 & 11 & 64 & 17 & 22 & 60 & & & \\
\hline$P$ value & \multicolumn{2}{|c|}{1.000} & \multicolumn{2}{|c|}{0.328} & \multicolumn{3}{|c|}{0.495} & \multicolumn{2}{|c|}{0.146} & \multicolumn{2}{|c|}{0.073} & & & \\
\hline \multicolumn{15}{|l|}{ pAkt } \\
\hline Negative & 66 & 4 & 63 & 7 & 55 & 9 & 6 & 64 & 4 & 28 & 39 & 48 & 15 & 7 \\
\hline Moderate & 99 & 7 & 88 & 17 & 58 & 30 & 17 & 87 & 17 & 34 & 71 & 40 & 22 & 37 \\
\hline Strong & 74 & 11 & 64 & 21 & 40 & 30 & 15 & 66 & 18 & 21 & 62 & 23 & 22 & 37 \\
\hline$P$ value & & & & & & 0.002 & & 0.0 & $1(+)$ & & 102 & $<0$ & $001(+)$ & \\
\hline
\end{tabular}

Fisher's exact test was used to obtain the $P$ values. $(+)$ and $(-)$, positive and negative correlations. Bold data represent significant values.

nodal status (0 vs. $\geq 1$ ), HER2 (negative vs. positive), pHER1 (negative vs. positive), pHER2 (negative and weak vs. strong), pHER3 (negative vs. positive), and HER4 (low vs. high). The results from the univariate Cox analysis are presented in Table 5; as expected, we found that high tumor grade, large tumor size and positive nodal status were significantly associated with poorer disease-free survival and overall survival. Also as anticipated, HER2 positivity was associated with poor disease-free survival; however, this positivity did not reach statistical significance in relation to overall survival $(P=0.06)$. For the other HER receptors, the Cox analysis confirmed the Kaplan-Meier plots with respect to disease-free survival, but interestingly we also found that strong pHER2 was highly associated with a reduction in overall survival. Likewise, a high amount of HER4 correlated with an increased period of overall survival. Lastly, we did not find expression of pErk or pAkt to be associated with survival in this patient series (data not shown). 
Protein expression levels in relation to clinicopathological properties

\begin{tabular}{|c|c|c|c|c|c|c|c|c|c|c|c|c|c|}
\hline & \multicolumn{3}{|c|}{ Tumor grade } & \multicolumn{3}{|c|}{ Tumor size } & \multicolumn{3}{|c|}{ Nodal status } & \multicolumn{2}{|c|}{ Recurrence } & \multicolumn{2}{|c|}{ Death } \\
\hline & 1 & 2 & 3 & $<2 \mathrm{~cm}$ & 2 to $5 \mathrm{~cm}$ & $>5 \mathrm{~cm}$ & 0 & 1 to 3 & $\geq 4$ & No & Yes & No & Yes \\
\hline \multicolumn{14}{|l|}{ HER2 } \\
\hline Negative & 61 & 96 & 49 & 78 & 149 & 19 & 22 & 137 & 87 & 169 & 77 & 137 & 109 \\
\hline Positive & 1 & 7 & 14 & 7 & 12 & 3 & 0 & 12 & 10 & 10 & 12 & 9 & 13 \\
\hline$P$ value & \multicolumn{3}{|c|}{$0.0003(+)$} & \multicolumn{3}{|c|}{0.506} & \multicolumn{3}{|c|}{0.335} & \multicolumn{2}{|c|}{$0.034(+)$} & \multicolumn{2}{|c|}{0.190} \\
\hline \multicolumn{14}{|l|}{ pHER1 } \\
\hline Negative & 50 & 82 & 49 & 62 & 136 & 19 & 16 & 125 & 76 & 150 & 67 & 121 & 196 \\
\hline Positive & 10 & 20 & 14 & 20 & 24 & 3 & 6 & 21 & 20 & 25 & 22 & 22 & 25 \\
\hline$P$ value & \multicolumn{3}{|c|}{0.731} & \multicolumn{3}{|c|}{0.188} & \multicolumn{3}{|c|}{0.180} & \multicolumn{2}{|c|}{$0.042(+)$} & \multicolumn{2}{|c|}{0.333} \\
\hline \multicolumn{14}{|l|}{ pHER2 } \\
\hline Negative & 43 & 57 & 24 & 49 & 92 & 14 & 12 & 88 & 55 & 105 & 50 & 91 & 64 \\
\hline Weak & 17 & 26 & 22 & 20 & 47 & 4 & 8 & 42 & 21 & 55 & 16 & 41 & 30 \\
\hline Strong & 1 & 19 & 17 & 12 & 22 & 4 & 2 & 16 & 20 & 16 & 22 & 12 & 26 \\
\hline$P$ value & \multicolumn{3}{|c|}{$0.0001(+)$} & \multicolumn{3}{|c|}{0.811} & \multicolumn{3}{|c|}{0.189} & \multicolumn{2}{|c|}{$0.001(+)$} & \multicolumn{2}{|c|}{$0.009(+)$} \\
\hline \multicolumn{14}{|l|}{ pHER3 } \\
\hline Negative & 50 & 88 & 50 & 69 & 134 & 18 & 20 & 123 & 78 & 153 & 68 & 122 & 99 \\
\hline Positive & 10 & 15 & 13 & 12 & 25 & 3 & 2 & 21 & 17 & 22 & 18 & 20 & 20 \\
\hline$P$ value & \multicolumn{3}{|c|}{0.582} & \multicolumn{3}{|c|}{1.000} & & 0.607 & & & 99 & & 506 \\
\hline HER4 & & & & & & & & & & & & & \\
\hline Low & 10 & 32 & 27 & 24 & 49 & 12 & 7 & 41 & 37 & 38 & 47 & 36 & 49 \\
\hline High & 51 & 67 & 35 & 56 & 109 & 10 & 13 & 105 & 57 & 133 & 42 & 104 & 71 \\
\hline$P$ value & & 0.004 & & & 0.088 & & & 0.174 & & & $01(-)$ & & $2(-)$ \\
\hline pErk & & & & & & & & & & & & & \\
\hline Negative & 20 & 45 & 35 & 32 & 72 & 9 & 12 & 57 & 44 & 76 & 37 & 63 & 50 \\
\hline Moderate & 13 & 25 & 11 & 18 & 40 & 3 & 4 & 33 & 24 & 42 & 19 & 32 & 29 \\
\hline Strong & 22 & 32 & 16 & 27 & 45 & 10 & 5 & 50 & 27 & 52 & 30 & 42 & 40 \\
\hline$P$ value & & 0.26 & & & 0.514 & & & 0.593 & & & 98 & & 317 \\
\hline pAkt & & & & & & & & & & & & & \\
\hline Negative & 10 & 29 & 20 & 20 & 46 & 4 & 8 & 33 & 29 & 48 & 22 & 42 & 28 \\
\hline Moderate & 30 & 34 & 26 & 38 & 59 & 9 & 9 & 54 & 43 & 64 & 42 & 58 & 48 \\
\hline Strong & 20 & 39 & 16 & 23 & 54 & 8 & 5 & 55 & 25 & 60 & 25 & 42 & 43 \\
\hline$P$ value & & 0.10 & & & 0.588 & & & 0.195 & & & 87 & & 419 \\
\hline
\end{tabular}

Fisher's exact test was used to obtain the $P$ values. $(+)$ and $(-)$, positive and negative correlations. Bold data represent significant values.

For the multivariate Cox regression we initially included all variables in a single model, which showed that only positive nodal status and high HER4 expression were significant predictors of decreased and prolonged survival, respectively (data not shown). This result is in agreement with the strong correlations between HER2, pHER1, pHER2 and pHER3, and therefore this analysis does not give a truthful picture of the importance of the three phosphorylated HER receptors. We therefore tested pHER1, pHER2 or pHER3 alone in a model containing the current standard of clinical biomarker parameters (that is, 
Figure 3
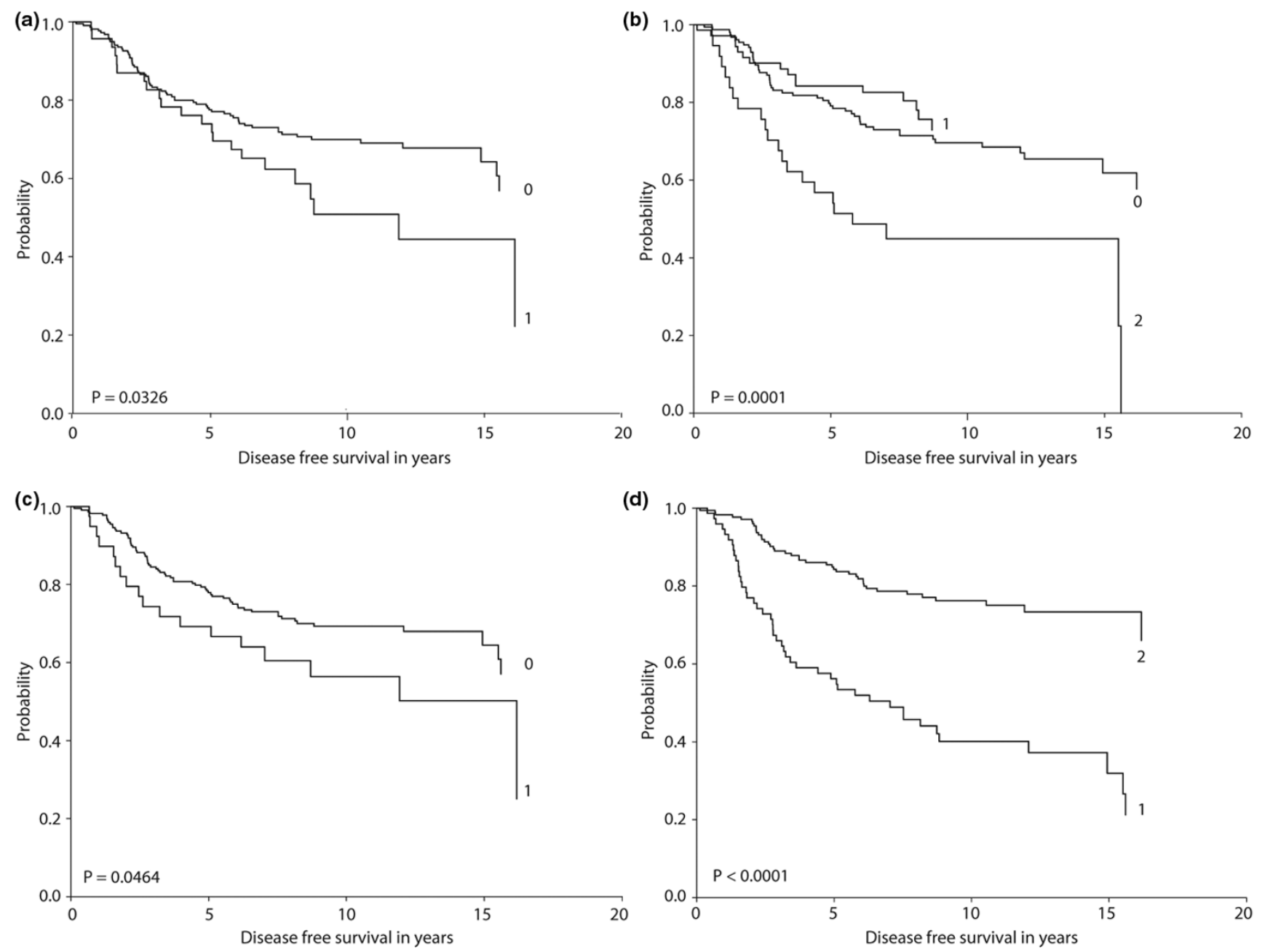

Kaplan-Meier curves showing disease-free survival in relation to protein expression levels. (a) pHER1: negative (0), positive (1). (b) pHER2: negative (0), weak (1), strong (2). (c) pHER3: negative (0), positive (1). (d) HER4: moderate (1), strong (2).

tumor grade, tumor size, nodal status and HER2). The results in Table 6 show that strong pHER2 expression was a significant and independent predictor of decreased disease-free survival. Furthermore, strong pHER2 expression highly significantly predicted for reduced overall survival, in contrast to the current HER2 analysis.

In this multivariate model, expression of pHER1 and pHER3 did not have independent prognostic value (data not shown).

\section{Comparison of frequencies of protein expression levels between 30 primary tumors and their corresponding metastases}

In addition to the primary tumor material, we also analyzed the protein expression levels of the six markers in 30 distant metastases from 30 patients within the original group. Table 7 presents the expression frequencies in the primary tumor and in the metastatic tumors. The final column in Table 7 presents the $P$ values from a Sign test showing that membrane expression of pHER1 and pHER3 as well as nuclear expression of pHER3 were significantly increased in the metastases. No difference in expression was observed with respect to pHER2. The pHER2 level in the 30 primary tumors was already markedly increased, however, compared with the average pHER2 expression level of the original 264 primary tumors. Lastly, the HER4 expression appeared to decrease, but this change was not statistically significant.

\section{Discussion}

The present study investigated the prognostic value of the activated HER1, HER2 and HER3 receptors, the total HER4 expression and the activated form of the downstream kinases Akt and Erk. We addressed this issue in a series of postmenopausal patients presenting with primary hormone receptorpositive breast cancer, who had all received adjuvant tamoxifen therapy. 
Table 5

Cox univariate regression analyses of variables in relation to disease-free survival and overall survival

\begin{tabular}{|c|c|c|c|c|}
\hline \multirow[t]{2}{*}{ Variable } & \multicolumn{2}{|c|}{ Disease-free survival } & \multicolumn{2}{|c|}{ Overall survival } \\
\hline & $P$ value & Relative risk $(95 \% \mathrm{Cl})$ & $P$ value & Relative risk $(95 \% \mathrm{Cl})$ \\
\hline Tumor grade & 0.0201 & 1.767 (1.093 to 2.86$)$ & 0.0072 & 1.785 ( 1.170 to 2.725$)$ \\
\hline Tumor size & 0.0007 & $2.070(1.357$ to 3.160$)$ & 0.0052 & 1.662 (1.164 to 2.375$)$ \\
\hline Nodal status & $<0.0001$ & 3.193 (2.091 to 4.876$)$ & $<0.0001$ & 2.205 (1.545 to 3.148$)$ \\
\hline HER2 & 0.0037 & $2.470(1.342$ to 4.546$)$ & 0.0595 & 1.739 (0.978 to 3.093$)$ \\
\hline pHER1 & 0.0346 & $1.683(1.038$ to 2.726$)$ & 0.2317 & 1.309 (0.842 to 2.033$)$ \\
\hline pHER2 & $<0.0001$ & $2.699(1.662$ to 4.384$)$ & 0.0002 & $2.273(1.470$ to 3.513$)$ \\
\hline pHER3 & 0.0489 & $1.686(1.002$ to 2.835$)$ & 0.3926 & 1.233 (0.763 to 1.995$)$ \\
\hline HER4 & $<0.0001$ & $0.361(0.238$ to 0.549$)$ & 0.0111 & $0.624(0.433$ to 0.898$)$ \\
\hline
\end{tabular}

Variables were analyzed as follows: tumor grade ( 1 and 2 vs. 3 ), tumor size $(<25 \mathrm{~mm}$ vs. $\geq 25 \mathrm{~mm}$ ), nodal status ( 0 vs. $\geq 1)$, HER2 (negative vs. positive), pHER1 (negative vs. positive), pHER2 (negative and weak vs. strong), pHER3 (negative vs. positive), HER4 (low vs. high). 95\% Cl, $95 \%$ confidence interval. Bold data represent significant values.

Total HER2 expression is an independent predictor of poor prognosis [2,7] and is also a clinical target for treatment [22]. In this study, $8 \%$ of the patients had HER2-positive tumors in accordance with this being a hormone receptor-positive population, and HER2 positivity was associated with shorter disease-free survival. Fourteen percent of cases were recorded with strong membrane expression of HER2 phosphorylated at tyrosine 1221/1222, however, and this HER2 phosphorylation was associated with high tumor grade and with shorter disease-free survival and overall survival. We speculate that the reason for this difference is due to the fact that moderate or even low levels of HER2 may be sufficient to elicit a potent mitogenic signal, upon activation by dimerization with ligand activated HER1 or HER3. This hypothesis is not new [23], and especially the importance of the HER ligands for activation of the HER receptors has been substantiated by several reports [3,24-26]. In agreement, we find that the hormone receptorpositive breast cancer cell line MCF-7, which we score HER2negative, has weak to moderate levels of HER2 mRNA and protein [27], and addition of the HER3/HER4 ligand heregulin $1 \beta$ clearly abrogated the inhibitory effect of antiestrogen treatment equally well in both wild-type and HER2/HER3 overexpressing MCF-7 cells - suggesting that activation of even a low level of HER2 via dimerization of ligand-activated HER3 may suffice to protect against antiestrogen therapy [27].

A recent study has compared HER2 mRNA expression in MCF-7 cells with mRNA expression in tumors classified as HER2-negative or HER2-positive, and the HER2 mRNA level in MCF-7 cells was lower than in most HER2-negative tumors [28]. This supports tumors classified as HER2-negative perhaps having sufficient HER2 protein to elicit signal transduction upon activation, and thereby may explain why also some patients with HER2-negative tumors respond to trastuzumab treatment [28].

It is noteworthy that $82 \%$ of cases with strong pHER2 staining without HER2 overexpression were scored positive for

Table 6

Cox multivariate regression analyses of selected variables in relation to disease-free survival and overall survival

\begin{tabular}{lllll}
\hline \multirow{2}{*}{ Variable } & \multicolumn{2}{l}{ Disease-free survival } & \multicolumn{2}{l}{ Overall survival } \\
& $P$ value & Relative risk $(95 \% \mathrm{Cl})$ & $P$ value & Relative risk (95\% Cl) \\
\hline Tumor grade & 0.544 & $1.17(0.71$ to 1.92$)$ & 0.187 & $1.35(0.87$ to 2.09$)$ \\
Tumor size & 0.018 & $1.76(1.10$ to 2.80$)$ & 0.060 & $1.48(0.98$ to 2.21$)$ \\
Nodal status & $<0.0001$ & $3.11(1.90$ to 5.11$)$ & 0.0001 & $2.29(1.50$ to 3.49$)$ \\
HER2 & 0.097 & $1.84(0.90$ to 3.78$)$ & 0.574 & $1.22(0.62$ to 2.40$)$ \\
pHER2 & 0.009 & $2.14(1.21$ to 3.78$)$ & 0.005 & $2.10(1.25$ to 3.52$)$
\end{tabular}

Variables were included in the following categories: tumor grade ( 1 and $2 \mathrm{vs.} 3$ ), tumor size ( $<25 \mathrm{~mm}$ vs. $\geq 25 \mathrm{~mm}$ ), nodal status (0 vs. $\geq 1)$, HER2 (negative vs. positive), pHER2 (negative and weak vs. strong). $95 \% \mathrm{Cl}, 95 \%$ confidence interval. Bold data represent significant values. 
Table 7

Frequencies of protein expression levels between primary and metastatic tumors

\begin{tabular}{|c|c|c|c|c|c|c|c|}
\hline \multirow[t]{2}{*}{ Protein } & \multicolumn{3}{|c|}{ Primary tumors } & \multicolumn{3}{|c|}{ Metastatic tumors } & \multirow[t]{2}{*}{ Sign test, $P$ value } \\
\hline & 0 & 1 & 2 & 0 & 1 & 2 & \\
\hline pHER1 & 25 & -- & 5 & 11 & -- & 19 & 0.0003 \\
\hline pHER2 & 17 & 5 & 7 & 14 & 10 & 6 & 0.6072 \\
\hline pHER3 & 23 & -- & 5 & 16 & -- & 13 & 0.0034 \\
\hline Nuclear pHER3 & 27 & -- & 1 & 15 & -- & 14 & 0.0002 \\
\hline HER4 & -- & 13 & 17 & -- & 19 & 10 & 0.1435 \\
\hline Cytoplasmic pErk & 10 & 4 & 15 & 6 & 12 & 12 & 1.0000 \\
\hline Nuclear pErk & 10 & 3 & 16 & 6 & 6 & 18 & 0.4545 \\
\hline Cytoplasmic pAkt & 3 & 19 & 8 & 10 & 5 & 14 & 0.8036 \\
\hline Nuclear pAkt & 16 & 8 & 8 & 10 & 7 & 12 & 0.0768 \\
\hline
\end{tabular}

Category $0=$ number of negative cases, category $1=$ number of weak-expressing (pHER2), low-expressing (HER4) or moderate-expressing (pErk and pAkt) cases, and category $2=$ number of positive (pHER1 and pHER3) or strong-expressing (pHER2, pErk and pAkt) cases. Expression of pHER1, pHER3 and nuclear pHER3 was analyzed as negative or positive, hence '--' in category 1 . HER4 expression was evaluated as either low or high, hence '--' in category 0 . The Sign test was used to examine for significant differences in protein expression levels between primary and metastatic tumors. Bold data represent significant values.

pHER1 and/or pHER3, clearly supporting that HER2 may be activated via dimerization with another HER family member. This is further supported by the finding that pHER2 was often expressed in the same tumor cells that were also positive for pHER1 and/or pHER3 (Figure 2). Our multivariate analysis clearly revealed that IHC evaluation of tyrosine 1221/1222 pHER2 was significantly better than the current HER2 tests with respect to select patients with both poor disease-free survival and overall survival. Other studies have also indicated that pHER2 expression may provide additional survival information $[23,29,30]$. In two of these studies, IHC detection of pTyr 1248 was measured and only a small fraction of the HER2positive cases $(12 \%)$ was positive for pHER2 $[23,29]$. In the study by Cicenas and colleagues [30], the same antibody against pTyr 1248 was applied in a highly sensitive chemoluminescence-linked immune assay. In that study, pHER2 expression was found in both HER2-positive and HER2-negative tumors (68\% and $27 \%$, respectively). In concert with our finding, the multivariate analysis showed that pHER2 was a marker of poor prognosis independent of HER2. We have tested the antibody against pTyr 1248 , but found only weak staining in our positive controls, indicating that this antibody may not be sensitive enough for IHC analysis as also suggested by Cicenas and colleagues [30]. We therefore selected the antibody against pTyr 1221/1222 directed against phosphotyrosines, which like pTyr 1248 is related to HER2 receptor activation [20]. Our data support that the pTyr1221/1222 antibody is a good antibody for IHC detection of activated HER2.

Future studies shall validate the clinical usefulness of the pTyr $1221 / 1222$ antibody with respect to predict response to endocrine therapy and also in relation to predict response to therapy directed against the activated HER2 receptor. Our finding that activated HER2 is expressed also in HER2-negative tumors indicates that these tumors may utilize HER2 receptor signaling to promote growth, and thus may be potential responders to treatment targeting the activated HER2. Besides trastuzumab, which has been found to be beneficial also in a fraction of the patients with HER2-negative tumors [28], targeted therapy may involve the monoclonal antibody Pertuzumab, which targets HER2 receptor dimerization [31], or treatment with the tyrosine kinase inhibitor Lapatinib, which targets the kinase activity of both HER2 and HER1 [32].

In the present series, membrane expression of phosphorylated HER3 at tyrosine 1289 was found in 15\% of cases and was associated with shorter disease-free survival. No previous work has investigated the importance of phosphorylated HER3 in primary breast cancers, but most studies of total HER3 expression have found HER3 to correlate with adverse clinicopathological properties [2,5,6,11,33,34]. Contrary results have also been observed, however; for example, inverse association with local recurrence [2] and association with longer survival $[13,14]$. In breast cancer cell lines, HER3 is a promoter of cell growth and is required for HER2-mediated proliferation [35]. More recently, HER3 activation has also been shown to be important for both tamoxifen-resistant and fulvestrant-resistant cell growth $[10,36]$, and a significant role for HER3 in resistance towards HER-directed therapy is also evident $[37,38]$. In the present patient series, membrane expression of pHER3 did not add prognostic information to the current clinical parameters, hence substantiating that 
HER2 is the dominant HER receptor and that the function of HER3 is primarily to act as a co-activator of HER2. Moreover, nuclear expression of pHER3 was found in 10\% of the patient tumors, but it was not significantly associated with the parameters investigated in the present series.

We observed membrane expression of HER1 phosphorylated at tyrosine 1173 in $18 \%$ of cases, and to the best of our knowledge only two other studies have measured pHER1 in breast cancer biopsies. These investigations were carried out on 225 and 154 cases of advanced breast cancer, and 7\% and 36\% of cases displayed membrane staining, respectively [39,40]. In agreement with these studies, our work showed that pHER1 was significantly correlated to shorter disease-free survival. Moreover, high total HER1 expression is also generally related to poor prognosis $[2,5,6]$. Overall, however, the literature on the prognostic value of total HER1 expression in breast cancer is not completely clear [41]. Our analyses show that membrane expression of pHER1 did not add prognostic value to the current clinical parameters, thus indicating that HER1, like HER3, also mainly acts as a co-activator of HER2.

In contrast to the predominantly membrane staining of total HER2, pHER1, pHER2 and pHER3, the HER4 staining was primarily cytoplasmic but also membranous. The overall intensity scoring method was therefore applied. HER4 expression was found in $97 \%$ of cases. The high percentage of positive cases is in concert with the data from Abd El-Rehim and coworkers, who found $80 \%$ HER4-positive cases in a series of more than 1,500 patients [2], and another study recorded 82\% HER4-positive cases [18]. When only reactivity in the membrane is scored, however, the observed frequencies are much lower: $21 \%$ [17], 14\% [5], and 12\% [6]. In agreement, we found $15 \%$ of cases to express HER4 in the membrane. In this series, high HER4 expression was inversely associated with pHER2 and tumor grade, and had a positive effect on disease-free survival and overall survival. Furthermore, high HER4 expression independently predicted for longer disease-free survival and overall survival, compared with the currently used parameters. This result is much in line with earlier data from both protein and mRNA analyses, where HER4 predicted prolonged survival in multivariate analysis $[6,14]$. Moreover, others have found associations between high HER4 expression and longer disease-free survival and/or overall survival compared with low or negative cases $[17,18]$.

We also looked at the activated levels of the HER downstream kinases Erk and Akt - we found that pAkt was correlated with pHER2, pHER3 and pErk, whereas pErk was not correlated with any of the HER receptors. Our data therefore suggest that pAkt is likely to be an important downstream mediator of HER2/HER3 signaling, which is very much in line with the current knowledge [8]. We did not find associations, however, between pAkt or pErk and clinicopathological parameters or survival. For pAkt this is in agreement with a study of 691 cases where the authors did not find an association with survival [42]. In contrast, others have observed pAkt expression in $54 \%$ of 93 patients analyzed and have shown that pAkt was an independent marker for disease-free survival [43]. Also, another study of 399 cases found pAkt to be associated with decreased overall survival in univariate analysis [44]. Few studies have been conducted for pErk, and also here conflicting results have been obtained. Using both immunoblotting and $\mathrm{IHC}$, it has been shown by multivariate analysis that pErk was a marker for prolonged survival [45], while others have reported that $\mathrm{pErk}$ independently predicted for reduced survival $[46,47]$. In our patient cohort, there was a trend for pErk to be associated with expression of HER4 $(P=0.07)$ and this would indicate a preferable effect of pErk, but this was not evident in the Cox analysis. Moreover, the number of pErk and pAkt targets is currently sought to be around 160 and 70 proteins, respectively $[48,49]$. This fact is likely to, at least partly, explain the discrepancies between the studies.

The comparison of the expression levels of the six proteins in 30 distant metastases and their corresponding primary tumors disclosed a significant increase in expression of pHER1 and pHER3 in the metastases. This observation fits well with the poor prognosis of cases with primary tumors expressing the phosphorylated receptors and, worthy of note, it indicates that pHER1 and pHER3 may be important activators of HER2 in metastatic tumor cell growth. We did not find other reports investigating pHER1, pHER2 and pHER3 levels in this setting, but eight other studies have, in agreement with our finding, consistently found that the total HER2 expression, evaluated by $\mathrm{IHC}$, is unaltered between the primary and metastatic lesions [50]. Analysis of activated HER1 and HER3 in distant metastasis may therefore be helpful in the clinical setting. Lastly, we observed a significant increase in the number of cases with nuclear expression of pHER3 in the metastasis. No data on nuclear HER3 expression exist in breast cancer, but a recent report showed that nuclear HER3 was absent in nonmalignant prostate tissue, whereas it was highly expressed in the cancerous prostate tissue and associated with increased tumor grade [51]. Studies with more statistical power should therefore investigate whether increased nuclear expression of HER3 in metastatic lesions may be associated with an adverse prognosis.

\section{Conclusions}

The present study shows that strong expression of activated HER2 as well as activated HER1 and HER3 is associated with poor prognosis in this series of hormone receptor-positive breast cancer patients. We conclude that expression of HER2 phosphorylated at tyrosine $1221 / 1222$ is likely to hold currently unassessed information on poor prognosis in hormone receptor-positive breast cancer.

\section{Competing interests}

The authors declare that they have no competing interests. 


\section{Authors' contributions}

$A-V L, T F$ and AEL were responsible for the study design. A-VL collected the samples and performed all IHC analyses. TF and $\mathrm{A}-\mathrm{VL}$ evaluated the $\mathrm{IHC}$ stainings. $\mathrm{MBL}$ provided the clinical patient data. $\mathrm{KLH}$ assisted with the $\mathrm{IHC}$ analysis and data interpretation. TF performed the statistical analysis, interpreted the data and drafted the manuscript. AEL critically revised the data interpretation and the manuscript, and obtained funding for the study. All authors revised the manuscript for important intellectual content.

\section{Acknowledgements}

The authors thank Ole Nielsen and Hanife Dzaferi for excellent technical assistance and Nick Martinussen for help with the statistical analyses. They much appreciate the financial support from the Danish Cancer Society, the Danish Medical Research Council and Købmand B. Rasmussen og hustru M. Rasmussens Mindelegat.

\section{References}

1. Citri A, Yarden Y: EGF-ERBB signalling: towards the systems level. Nat Rev Mol Cell Biol 2006, 7:505-516.

2. Abd El-Rehim DM, Pinder SE, Paish CE, Bell JA, Rampaul RS, Blamey RW, Robertson JF, Nicholson RI, Ellis IO: Expression and co-expression of the members of the epidermal growth factor receptor (EGFR) family in invasive breast carcinoma. $\mathrm{Br} J \mathrm{Can}$ cer 2004, 91:1532-1542.

3. Révillion F, Lhotellier V, Hornez L, Bonneterre J, Peyrat JP: ErbB/ HER ligands in human breast cancer, and relationships with their receptors, the bio-pathological features and prognosis. Ann Oncol 2008, 19:73-80.

4. Gee JM, Robertson JF, Gutteridge E, Ellis IO, Pinder SE, Rubini M, Nicholson RI: Epidermal growth factor receptor/HER2/insulinlike growth factor receptor signalling and oestrogen receptor activity in clinical breast cancer. Endocr Relat Cancer 2005, 12(Suppl 1):S99-S111.

5. Tovey S, Dunne B, Witton CJ, Forsyth A, Cooke TG, Bartlett JM: Can molecular markers predict when to implement treatment with aromatase inhibitors in invasive breast cancer? Clin Cancer Res 2005, 11:4835-4842.

6. Witton CJ, Reeves JR, Going JJ, Cooke TG, Bartlett JM: Expression of the HER1-4 family of receptor tyrosine kinases in breast cancer. J Patho/ 2003, 200:290-297.

7. Slamon DJ, Clark GM, Wong SG, Levin WJ, Ullrich A, McGuire WL: Human breast cancer: correlation of relapse and survival with amplification of the HER-2/neu oncogene. Science 1987, 235:177-182.

8. Hsieh AC, Moasser MM: Targeting HER proteins in cancer therapy and the role of the non-target HER3. Br J Cancer 2007, 97:453-457.

9. Hamburger AW: The role of ErbB3 and its binding partners in breast cancer progression and resistance to hormone and tyrosine kinase directed therapies. J Mammary Gland Biol Neoplasia 2008, 13:225-233.

10. Frogne T, Benjaminsen RV, Sonne-Hansen K, Sorensen BS, Nexo $E$, Laenkholm AV, Rasmussen LM, Riese DJ 2nd, de Cremoux $P$, Stenvang J, Lykkesfeldt AE: Activation of ErbB3, EGFR and Erk is essential for growth of human breast cancer cell lines with acquired resistance to fulvestrant. Breast Cancer Res Treat 2009, 114:263-275. Epub 2008 Apr 14.

11. Sassen $A$, Rochon J, Wild $P$, Hartmann A, Hofstaedter F, Schwarz S, Brockhoff G: Cytogenetic analysis of HER1/EGFR, HER2, HER3 and HER4 in 278 breast cancer patients. Breast Cancer Res 2008, 10:R2.

12. Xue C, Liang F, Mahmood R, Vuolo M, Wyckoff J, Qian H, Tsai KL, Kim M, Locker J, Zhang ZY, Segall JE: ErbB3-dependent motility and intravasation in breast cancer metastasis. Cancer Res 2006, 66:1418-1426.

13. Lee Y, Cho S, Seo JH, Shin BK, Kim HK, Kim I, Kim A: Correlated expression of erbB-3 with hormone receptor expression and favorable clinical outcome in invasive ductal carcinomas of the breast. Am J Clin Pathol 2007, 128:1041-1049.

14. Pawlowski V, Revillion F, Hebbar M, Hornez L, Peyrat JP: Prognostic value of the type I growth factor receptors in a large series of human primary breast cancers quantified with a real-time reverse transcription-polymerase chain reaction assay. Clin Cancer Res 2000, 6:4217-4225.

15. Naresh A, Long W, Vidal GA, Wimley WC, Marrero L, Sartor Cl, Tovey S, Cooke TG, Bartlett JM, Jones FE: The ERBB4/HER4 intracellular domain $4 I C D$ is a $\mathrm{BH} 3-$ only protein promoting apoptosis of breast cancer cells. Cancer Res 2006, 66:6412-6420.

16. Feng SM, Sartor $\mathrm{Cl}$, Hunter $\mathrm{D}$, Zhou H, Yang X, Caskey LS, Dy R, Muraoka-Cook RS, Earp HS III: The HER4 cytoplasmic domain, but not its $\mathrm{C}$ terminus, inhibits mammary cell proliferation. $\mathrm{Mol}$ Endocrinol 2007, 21:1861-1876.

17. Junttila TT, Sundvall M, Lundin M, Lundin J, Tanner M, Harkonen $P$, Joensuu $\mathrm{H}$, Isola J, Elenius $\mathrm{K}$ : Cleavable ErbB4 isoform in estrogen receptor-regulated growth of breast cancer cells. Cancer Res 2005, 65:1384-1393.

18. Suo Z, Risberg B, Kalsson MG, Willman K, Tierens A, Skovlund E, Nesland JM: EGFR family expression in breast carcinomas. $C$ erbB-2 and c-erbB-4 receptors have different effects on survival. J Pathol 2002, 196:17-25.

19. Hynes NE, Lane HA: ERBB receptors and cancer: the complexity of targeted inhibitors. Nat Rev Cancer 2005, 5:341-354.

20. Schulze WX, Deng L, Mann M: Phosphotyrosine interactome of the ErbB-receptor kinase family. Mol Syst Biol 2005, 1:2005.0008.

21. Henriksen KL, Rasmussen BB, Lykkesfeldt AE, Moller S, Ejlertsen $B$, Mouridsen HT: Semi-quantitative scoring of potentially predictive markers for endocrine treatment of breast cancer: a comparison between whole sections and tissue microarrays. J Clin Pathol 2007, 60:397-404.

22. Yeon $\mathrm{CH}$, Pegram MD: Anti-erbB-2 antibody trastuzumab in the treatment of HER2-amplified breast cancer. Invest New Drugs 2005, 23:391-409.

23. Thor AD, Liu S, Edgerton S, Moore D, Kasowitz KM, Benz CC, Stern DF, DiGiovanna MP: Activation (tyrosine phosphorylation) of ErbB-2 (HER-2/neu): a study of incidence and correlation with outcome in breast cancer. J Clin Oncol 2000, 18:3230-3239.

24. Atlas E, Cardillo M, Mehmi I, Zahedkargaran H, Tang C, Lupu R: Heregulin is sufficient for the promotion of tumorigenicity and metastasis of breast cancer cells in vivo. Mol Cancer Res 2003, 1:165-175.

25. Kong A, Calleja V, Leboucher P, Harris A, Parker PJ, Larijani B: HER2 oncogenic function escapes EGFR tyrosine kinase inhibitors via activation of alternative HER receptors in breast cancer cells. PLOS ONE 2008, 3:e2881.

26. Menendez JA, Mehmi I, Lupu R: Trastuzumab in combination with heregulin-activated Her-2 (erbB-2) triggers a receptorenhanced chemosensitivity effect in the absence of Her-2 overexpression. J Clin Oncol 2006, 24:3735-3746.

27. Larsen SS, Egeblad M, Jaattela M, Lykkesfeldt AE: Acquired antiestrogen resistance in MCF-7 human breast cancer sublines is not accomplished by altered expression of receptors in the ErbB-family. Breast Cancer Res Treat 1999, 58:41-56.

28. Paik $S, K i m ~ C$, Wolmark N: HER2 status and benefit from adjuvant trastuzumab in breast cancer. N Engl J Med 2008, 358:1409-1411.

29. DiGiovanna MP, Stern DF, Edgerton SM, Whalen SG, Moore D, Thor AD: Relationship of epidermal growth factor receptor expression to ErbB-2 signaling activity and prognosis in breast cancer patients. J Clin Oncol 2005, 23:1152-1160.

30. Cicenas J, Urban P, Kung W, Vuaroqueaux V, Labuhn M, Wight E, Eppenberger U, Eppenberger-Castori S: Phosphorylation of tyrosine 1248-ERBB2 measured by chemiluminescencelinked immunoassay is an independent predictor of poor prognosis in primary breast cancer patients. Eur J Cancer 2006, 42:636-645.

31. Agus DB, Gordon MS, Taylor C, Natale RB, Karlan B, Mendelson DS, Press MF, Allison DE, Sliwkowski MX, Lieberman G, Kelsey SM, Fyfe G: Phase I clinical study of pertuzumab, a novel HER dimerization inhibitor, in patients with advanced cancer. $J$ Clin Oncol 2005, 23:2534-2543. 
32. Spector NL, Xia W, Burris H III, Hurwitz H, Dees EC, Dowlati A, O'Neil B, Overmoyer B, Marcom PK, Blackwell KL, Smith DA, Koch KM, Stead A, Mangum S, Ellis MJ, Liu L, Man AK, Bremer TM, Harris J, Bacus S: Study of the biologic effects of lapatinib, a reversible inhibitor of ErbB1 and ErbB2 tyrosine kinases, on tumor growth and survival pathways in patients with advanced malignancies. J Clin Oncol 2005, 23:2502-2512.

33. Naidu R, Yadav M, Nair S, Kutty MK: Expression of c-erbB3 protein in primary breast carcinomas. $\mathrm{Br} J$ Cancer 1998, 78:1385-1390.

34. Travis A, Pinder SE, Robertson JF, Bell JA, Wencyk P, Gullick WJ, Nicholson RI, Poller DN, Blamey RW, Elston CW, Ellis IO: C-erbB3 in human breast carcinoma: expression and relation to prognosis and established prognostic indicators. $\mathrm{Br} J$ Cancer 1996, 74:229-233.

35. Holbro T, Beerli RR, Maurer F, Koziczak M, Barbas CF III, Hynes NE: The ErbB2/ErbB3 heterodimer functions as an oncogenic unit: ErbB2 requires ErbB3 to drive breast tumor cell proliferation. Proc Natl Acad Sci USA 2003, 100:8933-8938.

36. Liu B, Ordonez-Ercan D, Fan Z, Edgerton SM, Yang X, Thor AD: Downregulation of erbB3 abrogates erbB2-mediated tamoxifen resistance in breast cancer cells. Int J Cancer 2007, 120:1874-1882.

37. Zhou BB, Peyton M, He B, Liu C, Girard L, Caudler E, Lo Y, Baribaud F, Mikami I, Reguart N, Yang G, Li Y, Yao W, Vaddi K, Gazdar AF, Friedman SM, Jablons DM, Newton RC, Fridman JS, Minna JD, Scherle PA: Targeting ADAM-mediated ligand cleavage to inhibit HER3 and EGFR pathways in non-small cell lung cancer. Cancer Cell 2006, 10:39-50.

38. Sergina NV, Rausch M, Wang D, Blair J, Hann B, Shokat KM, Moasser MM: Escape from HER-family tyrosine kinase inhibitor therapy by the kinase-inactive HER3. Nature 2007, 445:437-441.

39. Nieto Y, Nawaz F, Jones RB, Shpall EJ, Nawaz S: Prognostic significance of overexpression and phosphorylation of epidermal growth factor receptor (EGFR) and the presence of truncated EGFRvIII in locoregionally advanced breast cancer. J Clin Oncol 2007, 25:4405-4413.

40. Magkou C, Nakopoulou L, Zoubouli C, Karali K, Theohari I, Bakarakos P, Giannopoulou I: Expression of the epidermal growth factor receptor (EGFR) and the phosphorylated EGFR in invasive breast carcinomas. Breast Cancer Res 2008, 10:R49.

41. Chan SK, Hill ME, Gullick WJ: The role of the epidermal growth factor receptor in breast cancer. J Mammary Gland Biol Neoplasia 2006, 11:3-11.

42. Panigrahi AR, Pinder SE, Chan SY, Paish EC, Robertson JF, Ellis IO: The role of PTEN and its signalling pathways, including AKT, in breast cancer; an assessment of relationships with other prognostic factors and with outcome. J Pathol 2004, 204:93-100.

43. Pérez-Tenorio G, Stal O: Activation of AKT/PKB in breast cancer predicts a worse outcome among endocrine treated patients. $\mathrm{Br} J$ Cancer 2002, 86:540-545.

44. Kirkegaard T, Witton CJ, McGlynn LM, Tovey SM, Dunne B, Lyon A, Bartlett JM: AKT activation predicts outcome in breast cancer patients treated with tamoxifen. J Pathol 2005, 207:139-146.

45. Milde-Langosch K, Bamberger AM, Rieck G, Grund D, Hemminger $\mathrm{G}$, Muller V, Loning T: Expression and prognostic relevance of activated extracellular-regulated kinases (ERK1/2) in breast cancer. Br J Cancer 2005, 92:2206-2215.

46. Gee JM, Robertson JF, Ellis IO, Nicholson RI: Phosphorylation of ERK1/2 mitogen-activated protein kinase is associated with poor response to anti-hormonal therapy and decreased patient survival in clinical breast cancer. Int J Cancer 2001, 95:247-254.

47. Adeyinka A, Nui Y, Cherlet T, Snell L, Watson PH, Murphy LC: Activated mitogen-activated protein kinase expression during human breast tumorigenesis and breast cancer progression. Clin Cancer Res 2002, 8:1747-1753.

48. Yoon S, Seger R: The extracellular signal-regulated kinase: multiple substrates regulate diverse cellular functions. Growth Factors 2006, 24:21-44.

49. Liu W, Bagaitkar J, Watabe K: Roles of AKT signal in breast cancer. Front Biosci 2007, 12:4011-4019.

50. Carlsson J, Nordgren $\mathrm{H}$, Sjostrom J, Wester K, Villman K, Bengtsson NO, Ostenstad B, Lundqvist H, Blomqvist C: HER2 expres- sion in breast cancer primary tumours and corresponding metastases. Original data and literature review. $\mathrm{Br} J$ Cancer 2004, 90:2344-2348.

51. Koumakpayi IH, Diallo JS, Le PC, Lessard L, Filali-Mouhim A, Begin LR, Mes-Masson AM, Saad F: Low nuclear ErbB3 predicts biochemical recurrence in patients with prostate cancer. $B J U$ Int 2007, 100:303-309. 